\title{
Comparison of Two Specimen-Collection and Microscopy Methods for the Rapid Laboratory Diagnosis of Seborrheic Dermatitis- A Pilot Study
}

\author{
Harshita Hemant Sisodiya ${ }^{1}$, Nayana Avinash Ingole², Shashir Wanjare³, Uday S. Khopkar, Preeti Rajeev Mehta ${ }^{5}$
}

\begin{abstract}
${ }^{1}$ Department of Microbiology, Seth GS Medical College and KEM Hospital, Mumbai, Maharashtra, India. ${ }^{2}$ Department of Microbiology, Seth GS Medical College and KEM Hospital, Mumbai, Maharashtra, India. ${ }^{3}$ Department of Microbiology, Seth GS Medical College and KEM Hospital, Mumbai, Maharashtra, India. ${ }^{4}$ Department of Dermatology, Seth GS Medical College and KEM Hospital, Mumbai, Maharashtra, India. ${ }^{5}$ Department of Microbiology, Seth GS Medical College and KEM Hospital, Mumbai, Maharashtra, India.
\end{abstract}

\section{ABSTRACT}

\section{BACKGROUND}

Malassezia spp. causes seborrheic dermatitis. For laboratory diagnosis, skin scrapings are collected and mounted in potassium hydroxide (KOH) for microscopy and processed for culture. Obtaining scrapings has disadvantages and $\mathrm{KOH}$ lacks colour contrast making interpretation difficult. This pilot study compared the results of specimen collection by cellophane tape method with scraping method. It also compared microscopy using Chicago Sky Blue 6B (CSB) stain plus KOH with the conventional method using $\mathrm{KOH}$ alone.

\section{METHODS}

Skin specimens were collected from the affected sites of 80 patients by scraping and cellophane tape. Specimens were subjected to KOH examination, KOH plus CSB stain, and culture for the presence of Malassezia spp.

\section{RESULTS}

A total of 160 specimens were collected from 80 patients for microscopy. Of 160 specimens, each was subjected to KOH and CSB plus KOH, 145 (91\%) demonstrated Malassezia spp. by CSB plus KOH and $124(77.5 \%)$ by KOH alone ( $\mathrm{p}=0.001)$. Cellophane tape method yielded 141 (88\%) positive results compared to 128 (80\%) by skin scraping $(\mathrm{p}=0.047)$. The odds of detecting Malassezia spp. was 4.4 times greater when the specimen was collected by cellophane tape and subjected to microscopy with CSB and KOH than when it was collected by scraping and examined microscopically with $\mathrm{KOH}$ alone $(\mathrm{p}=0.002)$.

\section{CONCLUSIONS}

Cellophane tape is a convenient method for specimen collection. CSB stain provides colour contrast and enables easy identification of fungal elements.

\section{KEY WORDS}

Cellophane Tape Collection, Chicago Sky Blue 6B Stain, Seborrheic Dermatitis
Corresponding Author: Dr. Nayana Avinash Ingole, Associate Professor, Seth Gordhandas Sundardas Medical College, and King Edward Memorial Hospital, Parel, Mumbai-400012, Maharashtra, India.

E-mail: nayanaingole@gmail.com

DOI: $10.14260 / \mathrm{jemds} / 2020 / 207$

Financial or Other Competing Interests: None.

How to Cite This Article:

Sisodiya HH, Ingole NA, Wanjare $S$, et al. Comparison of two specimen-collection and microscopy methods for the rapid laboratory diagnosis of seborrheic dermatitis- a pilot study. J. Evolution Med. Dent. Sci. 2020;9(12):961-964, DOI: 10.14260/jemds/2020/207

Submission 16-01-2020,

Peer Review 25-02-2020,

Acceptance 04-03-2020,

Published 23-03-2020.

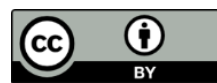




\section{BACKGROUND}

Malassezia yeasts have been recognized as members of normal human cutaneous flora as well as agents of certain skin diseases. They are lipophilic organisms and have frequently been found on a diverse range of other warmblooded animals. Malassezia genus is classified in the order Malasseziales among the Exobasidiomycetes. Characteristic features of the genus Malassezia include a distinctive morphology and an affinity for lipids in culture. Malassezia is implicated in mild but often recurrent cutaneous infections and also have been associated with skin and deep invasive infections in immunocompromised patients. Genus Malassezia comprises of 15 species of "yeast like fungi," 13 of which are lipophilic and 1 is non-lipophilic. They are $M$. furfur, M. pachydermatis, M. sympodialis, M. globosa, M. obtusa, $M$. restricta, $M$. slooffiae, M. equina, $M$. dermatis, $M$. japonica, M. nana, M. capre, M. yamatoensis, and most recently $M$. cuniculi, and Malassezia arunalokei species.[1-2]

Seborrheic dermatitis (SD) is the second most common pathology associated with Malassezia spp. and its prevalence in the general population is estimated to be $5 \%$. It is a common chronic inflammatory superficial eczematous dermatitis, either sub-acute or chronic, characterized by erythematous plaques with dry or oily scales. This condition is more common in males than in females. Any age is affected especially teenagers and adults. It is seen over areas rich in sebaceous glands-namely, the scalp, face, chest, back and flexural areas. A milder variant is dandruff, which is manifested by dry, flaking scales on the scalp. [1-2]

The mycological study is usually done by potassium hydroxide $(\mathrm{KOH})$ mount and culture. However, both have their own limitations. Samples for diagnosis are usually collected by direct scraping of skin which is time consuming and sometimes uncomfortable for patients. However, obtaining skin scraping is time-consuming, requires the use of a sharp instrument with its attendant concerns and risk of injury, limits the amount of material collected, and sensitivity and specificity are low. Collection of specimens using a cellophane tape is a useful alternative. ${ }^{[3-5]}$

Diagnosis by culture is time-consuming with inconsistent sensitivity.[6] Direct examination of $10 \%$ potassium hydroxide $(10 \% \mathrm{KOH})$ mount of the specimen is simpler and provides a faster diagnosis but has major drawbacks: it lacks sensitivity, there is interference by artefacts, it requires expertise and is time-consuming. ${ }^{[6-7]}$ This is chiefly due to the lack of contrast. Chicago Sky Blue 6B (CSB), a direct diazo dye compound, is used as a contrast in various pathology and ophthalmology procedures, and its usefulness has been reported in the diagnosis of pityriasis versicolor. It makes it easy to locate the fungal elements even under scanner view. Another advantage over $\mathrm{KOH}$ mount is that it outlines the morphological features of both the spores and the hyphae and can enable detailed study of fungal morphology under oil immersion microscopy. ${ }^{[8]}$

This pilot study aimed to compare specimen collection by cellophane tape method and subsequent microscopy by CSB stain and $\mathrm{KOH}$ with the routine collection method by scraping and microscopy by $\mathrm{KOH}$ alone.

\section{METHODS}

This cross-sectional pilot study was carried out at a tertiary care centre over a period of six months. Institutional and ethical approvals were obtained. Given a prevalence of seborrheic dermatitis of $5 \%$ in the general population ${ }^{[2]}$ and taking a confidence level of $95 \%$ and power of $80 \%$, a sample size of 80 was derived for this pilot study. Therefore 80 consecutive adult patients (age $\geq 18$ years) clinically suspected of having seborrheic dermatitis and presenting to the dermatology clinic during the study period were included. Patients who had been treated with oral or topical antifungal agents in the past one month were excluded.

Specimens were collected by scraping and cellophane tape, one each for $\mathrm{KOH}$ mount, $\mathrm{KOH}$ and CSB mount, and were cultured on Sabouraud's dextrose agar (SDA) with chloramphenicol and gentamicin overlaying with sterile olive oil. Specimens collected by scraping and cellophane tape were inoculated on tubes and plates of SDA respectively.

\section{Scraping Method}

After cleaning the affected area with 70\% isopropyl alcohol, skin scrapings were taken from the edge of the lesion with the blunt edge of a sterile scalpel blade (no. 10). Scrapings were placed on two clean microscopy slides, one of which had three drops of $10 \% \mathrm{KOH}$ solution, and the other $10 \% \mathrm{KOH}$ with CSB stain (Sigma-Aldrich, USA). CSB stain was prepared by dissolving $1 \mathrm{gm}$ CSB 6B powder (Sigma-Aldrich, USA) in $100 \mathrm{ml}$ distilled water. For culture, specimens were collected on a sterile Whatman filter paper.

\section{Cellophane Tape Method}

Two $5 \mathrm{~cm} \mathrm{x} 2 \mathrm{~cm}$ strips of clear transparent cellophane tape (Wonder Tape 555, Wonder Tape Industries, India) were applied over the affected site, pressed firmly, removed. Then they were then stuck on the surface of two clean microscopy slides, one with $10 \% \mathrm{KOH}$ solution, and the other with $10 \%$ KOH plus CSB stain. Specimens for culture were taken from the skin by stripping with a third $1 \mathrm{~cm} \times 1 \mathrm{~cm}$ strip of tape.

\section{Microscopy}

The slides were visualized under low power (10x magnification) as well as high power (40x magnification). The presence of hyphae and spores exhibiting characteristic "spaghetti and meatballs" appearance was considered positive for the presence of Malassezia spp. ${ }^{[9]}$

\section{Culture}

Specimens collected by scraping and cellophane tape were inoculated on tubes and plates respectively of Sabouraud's dextrose agar (SDA) with chloramphenicol and gentamicin (HiMedia; Mumbai, India). They were overlaid with sterile olive oil and they were incubated at $32^{\circ} \mathrm{C}$ for seven days. ${ }^{[9-10]}$ Growth of Malassezia spp. was confirmed by colony morphology, urease, catalase tests and safranin staining. ${ }^{[9,10]}$

\section{Statistical Analysis}

Data was analysed using SPSS (v16.0; SPSS Inc; Chicago, USA). Descriptive statistics, median and percentages, were calculated to describe demographic data. The categorical 
variables were compared using Pearson's $\chi^{2}$ test or McNemar test when appropriate. $\mathrm{p}$ value $\leq 0.05$ was considered significant.

Time to detection in positive specimens was calculated from the moment the specimen was collected by cellophane tape or scalpel to the moment characteristic morphology was observed.

\section{RESULTS}

Of the 80 patients who were included, 46 (58\%) were in the age group 18-30 years. Median age was 29 years. Forty-six patients $(58 \%)$ were males and 34 were females. Male to female ratio was 1.35:1 with a slight male preponderance. Five seborrheic dermatitis patients reported exposure to sunlight as a predisposing factor for seborrheic dermatitis. No other history of allergies to oil, talcum powder, shampoos or predisposing factors like temperature or heat difference could be elicited. The most common site affected in SD patients was scalp, followed by neck, back, forehead and nose. All patients $(100 \%)$ had scaly lesions, $64(80 \%)$ had itchy lesions and 67 (84\%) had hypo-pigmented lesions. The commonly affected sites were scalp (60\%), neck (45\%), back (33\%), forehead (28\%) and nose (25\%).

Malassezia spp. was detected by microscopy in $73(91 \%)$ patients, and by culture in $35(44 \%)$ patients $(\mathrm{p}<0.001)$. The difference between microscopy and culture for detecting Malassezia, was statistically significant. Of 160 specimens collected by each method, Malassezia spp. was detected in 141 (88\%) collected by cellophane tape and in 128 (80\%) collected by scraping ( $\mathrm{p}=0.047)$. Malassezia spp. was detected in $145(91 \%)$ of 160 specimens mounted in $\mathrm{KOH}$ with CSB, compared to $124(78 \%)$ of 160 mounted in $\mathrm{KOH}$ alone ( $\mathrm{p}=$ 0.001). The odds of detecting Malassezia spp. was 4.4 times greater when the specimen was collected by cellophane tape and subjected to microscopy with CSB and $\mathrm{KOH}$ than when it was collected by scraping and examined microscopically with $\mathrm{KOH}$ alone ( $\mathrm{p}=0.002)$ (table 1$)$. The median time to detection in positive specimens for collection by cellophane tape method and microscopic examination CSB and $\mathrm{KOH}$ was two minutes, whilst that for collection by scraping and examination by $\mathrm{KOH}$ was five minutes.

\begin{tabular}{|cccc|}
\hline Method & Positive & Negative & Total \\
Cellophane tape \& CSB & $73(91 \%)$ & $7(9 \%)$ & 80 \\
Scalpel \& KOH & $56(70 \%)$ & $24(30 \%)$ & 80 \\
\hline Table 1. Comparison of the & Two Collection & and Staining & Methods \\
\hline
\end{tabular}

\section{DISCUSSION}

Seborrheic dermatitis (SD) is a common, relapsing dermatitis affecting sebum-rich areas of the skin and is caused by fungi of the Malassezia spp. It is a common, relapsing dermatitis that is characterized by erythematous patches and superficial scaling. It affects sebaceous-rich gland areas - namely the scalp, face, central chest and anogenital areas. Adult seborrheic dermatitis is reported to involve one or more sites which includes scalp, eyebrows, forehead, nasal alar creases, chest and genital region. [9]
Seborrheic dermatitis is a chronic, recurrent condition, and patients generally experience frequent relapses. Usually seborrheic dermatitis is mild and responsive to therapy. But in severe or atypical cases, a variety of other entities should be considered in the differential diagnosis. The clinical presentation, though very typical, may be confused with other skin disorders like psoriasis, atopic dermatitis, contact dermatitis, and erythrasma and treatment for each differs. The diagnosis of seborrheic dermatitis is based on typical clinical picture, positive direct microscopy and culture. Isolation of Malassezia spp. from clinical specimens remains a challenge because of their lipid dependency, making culture a time-consuming exercise with inconsistent results and a low sensitivity. The cultures are considered positive for Malassezia if there is growth of cream to yellowish brown colonies, confirmed by morphological and biochemical tests. False negative findings in culture may arise when specimen contains dead or nonviable mycotic organisms or when the specimen is not enough. Therefore, as compared to culture, direct microscopic examination of specimens could be considered as the simplest and most inexpensive method for the laboratory diagnosis of Malassezia. [6-7]

Seborrheic dermatitis should always be confirmed with direct microscopy or culture to demonstrate pseudohyphae and blastoconidia in typical "spaghetti and meatballs" pattern. Direct microscopic examination of specimens is the simplest and most inexpensive method for the laboratory diagnosis of Malassezia spp. Direct examination of skin scraping by $\mathrm{KOH}$ examination is done for demonstration of fungal spores and hyphae. But this needs expertise as there is no contrast. [6-7] Skin scrapings from the affected area are the usual specimens submitted for microscopy. However, this method has disadvantages which affect the sensitivity and specificity of microscopy: only a relatively small area of the skin can be sampled, quantity of specimen collected is less, and microscopy of skin scrapings is compounded by the presence of artefacts (especially due to crystallisation of $\mathrm{KOH}$ or "ferning"). Additionally, the method demands manual dexterity, is time-consuming and there is risk of injury to the patient and patient concern arising from the use of a sharp instrument like a scalpel blade.[11-12]

Cellophane tape method was first used for the diagnosis of tinea versicolor and later its superiority was demonstrated for the diagnosis of dermatophytosis and superficial candidiasis.[4,13-15]Miranda et al.[12] reported a diagnostic sensitivity of $98.3 \%$ for superficial mycoses. In the present study, the diagnostic yield of Malassezia spp. from specimens collected by cellophane tape was significantly greater than that from scrapings. Cello tape method is also a relatively safer option for specimen collection and is suitable for the collection of sufficient quantity of skin specimen and is particularly useful if the examiner is inexperienced. Besides being a safer method than scraping, cellophane tape collection enables sampling of a larger area, preserves fungal morphology better and prevents clumping, thereby increasing sensitivity. In addition, it has excellent sensitivity \& accuracy of examination and the fungal hyphae are minimally distorted by this technique.[3,11] Also, this is a simpler method requiring no special dexterity or training, takes less time and facilitates transportation and storage.[13,16] It is also effective for direct microscopic diagnosis of chromomycosis, lobomycosis, and para- 
coccidioidomycosis, Pityriasis versicolor, dermatophytosis, Enterobius vermicularis etc. But it has few disadvantages like specimen collection from moist highly inflamed areas is difficult and excessive handling of the sticky surface of the tape or the presence of oil or grease may lead to opacification of the tape.[16]

Various contrast agents have been evaluated for the diagnosis of superficial mycoses. Chlorazol-KOH testing has low sensitivity and specificity and may be carcinogenic, whilst acridine orange and calcofluor white require fluorescent microscopy.[15,17] Chicago Sky Blue 6B (CSB) is a diazo dye generally used as a counterstain in immunofluorescence histochemistry, to assess viability of spermatozoa in animal studies and for dye-assisted macular surgery. CSB stain was used by Lodha et al, for rapid diagnosis of pityriasis versicolor by providing a colour contrast. [8] Several workers have reported increased sensitivity and specificity of microscopy using CSB in the diagnosis of dermatophytosis and superficial candidiasis. $[8,15,17,18] \quad$ In the present study, we found that addition of $\mathrm{CSB}$ to $\mathrm{KOH}$ provided a colour contrast that made reading and interpretation of slides, easier and took lesser time to interpret. It highlighted morphological features of fungi, superiorly. It was easy to perform and required only an ordinary light microscope and hence can be a good a cost effective, clinic-based test for rapid diagnosis of seborrheic dermatitis. CSB stain significantly increased the diagnostic yield for seborrheic dermatitis over a plain $\mathrm{KOH}$ mount.

Our study has a few drawbacks. This was a pilot study with the methods being assessed on the same lesions from the same patients with no randomisation or blinding. A larger randomised controlled study with blinding as well as a cost assessment would be more conclusive.

\section{CONCLUSIONS}

Specimen collection by cellophane tape increased the diagnostic yield of Malassezia spp. and was easier than scrapings. Microscopy with CSB stain significantly increased the diagnostic yield for seborrheic dermatitis over microscopy with plain $\mathrm{KOH}$ mount.

\section{REFERENCES}

[1] Thayikkannu AB, Kindo AJ, Veeraraghavan M. Malassezia spp. Can it be ignored? Indian J Dermatol 2015;60(4):332-9.

[2] Chatzikokkinou P, Sotiropoulos K, Katoulis A, et al. Seborrheic dermatitis-an early and common skin manifestation in HIV patients. Acta Dermatovenerol Croat 2008;16(4):226-30.

[3] Hassab-El-Naby HM, Salem AS, Abdo HM, et al. Comparative study for the reliability of cellophane tape and standard $\mathrm{KOH}$ mount in diagnosis of pityriasis versicolor. Global J Dermatol Venereol 2010;17(2):2934.
[4] Thirumurthy M, Sethuraman G, Srinivas CR. KOH mount for superficial fungal infections using cellophane tape: comparison with standard technique. Indian J Dermatol Venereol Leprol 2002;68(3):136.

[5] Miranda MFR, Silva AJG. Vinyl adhesive tape also effective for direct microscopy diagnosis of chromomycosis, lobomycosis, and paracoccidioidomycosis. Diagn Microbiol Infect Dis 2005;52(1):39-43.

[6] Jung MY, Shim JH, Lee JH, et al. Comparison of diagnostic methods for onychomycosis and proposal of a diagnostic algorithm. Clin Exp Dermatol 2015;40(5):479-84.

[7] Weinberg JM, Koestenblatt EK, Tutrone WD, et al. Comparison of diagnostic methods in the evaluation of onychomycosis. J Am Acad Dermatol 2003;49(2):193-7.

[8] Lodha N, Poojary SA. A novel contrast stain for the rapid diagnosis of pityriasis versicolor: a comparison of Chicago Sky Blue 6B stain, potassium hydroxide mount and culture. Indian J Dermatol 2015;60(4):340-4.

[9] Midgley G, Gueho E, Guillot J. Disease caused by Malassezia species. In: Merz W, Hay RJ. eds. Topley and Wilson's Medical Mycology. 10 th edn. New York: John Wiley and Sons Ltd., 1998;4:201-11.

[10] Wikler JR, De Haan P, Nieboer C. The 'tape-method': a new and simple method for quantitative culture of Pityrosporum yeasts. Acta Derm Venereol 1988;68(5):445-9.

[11] Remya VS, Arun B, Sheeba PM, et al. A study on Malassezia Micro Flora in the skin of healthy individuals in North Kerala, India. Int J Res Med Sci 2017;5(10):4600-3.

[12] Miranda MFR, Silva AJG. New uses of vinyl tape for reliable collection and diagnosis of common superficial mycoses. Skinmed 2003;2(3):156-8.

[13] Porto JA. The use of cellophane tape in the diagnosis of tinea versicolor. J Invest Dermatol 1953;21(4):229-31.

[14] Milne LR, Barnetson RC. Diagnosis of dermatophytosis using vinyl adhesive tape. Sabouraudia 1974;12(2):1625.

[15] Lim CSH, Lim SL. New contrast stain for the rapid diagnosis of dermatophytic and Candidal dermatomycoses. Arch Dermatol 2008;144(9):1228-9.

[16] Raghukumar S, Ravikumar BC. Potassium hydroxide mount with cellophane adhesive tape: a method for direct diagnosis of dermatophyte skin infections. Clin Exp Dermatol 2018;43(8):895-8.

[17] Prakash R, Prashanth HV, Ragunatha S, et al. Comparative study of efficacy, rapidity of detection, and cost-effectiveness of potassium hydroxide, calcofluor white, and Chicago sky blue stains in the diagnosis of dermatophytoses. International Journal of Dermatology 2016;55(4):e172-5.

[18] Kamath N, Swaminathan R, Sonawane J, et al. Comparison of rapid fungal staining technique $1 \%$ Chicago blue 6B stain for the diagnosis of superficial fungal infections (Pityriasis Versicolor/ Tinea Versicolor) with $10 \% \mathrm{KOH}$ mount and routine fungal culture on Sabouraud's dextrose agar with Olive oil overlay. J Med Sci Clin Res 2016;4(11):13600-3. 